\title{
Warm molecular gas, dust and ionized gas in the 500 central pc of the Galaxy
}

\author{
N.J. Rodríguez-Fernández ${ }^{* 1}$, J. Martín-Pintado ${ }^{2}$, A. Fuente ${ }^{3}$, and T.L. Wilson ${ }^{4}$ \\ ${ }^{1}$ LERMA, Observatoire de Paris, 61, Av de l'Observatoire, 75014 Paris, France \\ ${ }^{2}$ Instituto de Estructura de la Materia, CSIC, Serrano 121, 28006, Madrid, Spain \\ ${ }^{3}$ Observatorio Astronómico Nacional, IGN, Apdo. 1143, 28800 Alcalá de Henares, Spain \\ ${ }^{3}$ Max-Planck-Institut für Radioastronomie, Auf dem Hügel 69, 53121 Bonn, Germany
}

Received 15 November 2002, revised 30 November 2002, accepted 2 December 2002

Published online 3 December 2002

Key words ISM: lines - ISM: Infrared - Galaxy: center

PACS 04A25

We present infrared and millimeter observations of molecular gas, dust and ionized gas towards a sample of clouds distributed along the 500 central pc of the Galaxy. The clouds were selected to investigate the physical state, in particular the high gas temperatures, of the Galactic center region (GCr) clouds located far from far-infrared of thermal radio continuum sources. We have found that there is ionized gas associated with the molecular gas. The ionizing radiation is hard $(\sim 35000 \mathrm{~K})$ but diluted due to the inhomogeneity of the medium. We estimate that $\sim 30 \%$ of the warm molecular gas observed in the GCr clouds is heated by ultra-violet radiation in photo-dissociation regions.

\section{Introduction}

The interstellar medium in the 500 central pc of the Galaxy (hereafter Galactic Center region, GCr) is mainly molecular. The molecular clouds in the GCr exhibit an extended gas component with high temperature $(150 \mathrm{~K})$. On the contrary, the dust temperature is lower than $\sim 30 \mathrm{~K}$. The large line widths of the molecular lines, the high gas phase abundance of molecules linked to the dust chemistry, and the difference between gas and dust temperature suggest that some kind of shocks could be responsible for the high gas temperatures of the molecular gas (Wilson et al. 1982, Martín-Pintado et al. 2001). The possible influence of radiation in the heating of the molecular gas is also usually ruled out due to the lack of far infrared and thermal radio continuum sources in the GCr others than the well known H II regions associated to the Sgr complexes $(\mathrm{A}, \mathrm{B}, \ldots)$ or ionized nebulae like the Sickle.

To investigate the heating of the molecular clouds in the $\mathrm{GCr}$ we have studied a sample of 18 clouds located all along this region. The clouds were selected as molecular peaks located far from far infrared or radio continuum sources. Those sources were observed with the spectrometers on board the Infrared Space Observatory (ISO) and with the IRAM 30-m telescope. The data obtained with ISO are observations of the lowest $\mathrm{H}_{2}$ pure-rotational lines, dust continuum spectra from 40 to $190 \mu \mathrm{m}$ and a number of fine-structure lines from neutral atoms or ions (O I $63 \mu \mathrm{m}$, C II $158 \mu \mathrm{m}$, Ne II $12 \mu \mathrm{m}$, O III $52 \mu \mathrm{m}$...). With the IRAM 30-m antenna we have observed $\mathrm{C}^{18} \mathrm{O},{ }^{13} \mathrm{CO}, \mathrm{H} 35 \alpha$ and $\mathrm{H} 41 \alpha$.

In these paper we will review the results already published in Rodríguez-Fernández et al. (2001a, 2001b, hereafter RF01a, RF01b, respectively) and we will present some new results that will be extended elsewhere (Rodríguez-Fernández et al. 2003, RF03).

* e-mail: nemesio.rodriguez@obspm.fr, Phone: +33 140512 061, Fax: +33 140512002 


\section{Warm molecular gas}

Before ISO, the warm gas component of the GCr molecular clouds was mainly studied by means of $\mathrm{NH}_{3}$ observations. The multilevel study of Huttemeister et al. (1993) showed that the temperature structure of the $\mathrm{GCr}$ clouds can be characterized with two gas components at different temperatures: a cold gas component with a temperature close to that of the dust $(\sim 20 \mathrm{~K})$ and a warm gas component whose temperature ranges from 100 to $250 \mathrm{~K}$. However, since the abundance of ammonia is known to vary significantly is difficult to estimate the column density of warm gas in the $\mathrm{GCr}$ clouds. We have, for the first time, obtained the column density of warm gas in the $\mathrm{GCr}$ clouds by observing $\mathrm{H}_{2}$ pure rotational lines (RF01a). Columns 3 and 4 of Table 1 show the total column density and the temperature of the warm gas, respectively. We have also estimated the $\mathrm{H}_{2}$ density and the total column density of molecular gas in this clouds by observing ${ }^{13} \mathrm{CO}$ and $\mathrm{C}^{18} \mathrm{O}$ and doing a radiative transfer analysis for kinetic temperatures between 15 and $200 \mathrm{~K}$. The results are shown in columns 1 and 2 of Table 1 . The fraction of warm $\mathrm{H}_{2}$ to the total $\mathrm{H}_{2}$ column density as traced by $\mathrm{CO}$ varies from source to source but it is of $30 \%$ on average.

As discussed in RF01a, it is difficult to explain the large column density of warm gas in the $\mathrm{GCr}$ clouds. Several low velocity $\left(\sim 10 \mathrm{Km} \mathrm{s}^{-1}\right)$ C-shocks, photo-dissociation regions (PDRs) or both should be present in the line of sight. Comparing the energy of the turbulent motions in the GCr clouds with the cooling by $\mathrm{H}_{2}$ (which at the moderate density of the $\mathrm{GCr}$ clouds is comparable to that by $\mathrm{CO}$ ), one finds that dissipation of supersonic turbulence could account for the heating of the warm $\mathrm{H}_{2}$. However, with the available data is not possible to rule out heating in PDRs, indeed the observed temperature gradient in the GCr clouds can be appropriately reproduced in a context of a PDR (RF01a).

\section{Dust temperatures}

The dust continuum emission peaks at wavelengths of 100 to $80 \mu \mathrm{m}$ for all but two sources, whose spectra peaks at 50-60 $\mu \mathrm{m}$. The dust luminosity in the observed range is listed in column 5 of Table 1. It is not possible to fit the spectra with just one grey body. Thus we have used a model with two grey bodies like that described in section 2 of Goicoechea et al. (2003). Figure 1 shows the data and the grey bodies fits for two sources. To explain the emission at long wavelengths it is needed a dust component with a temperature of 15 to $18 \mathrm{~K}$. The temperature of the warmer component varies from source to source from 26 to $39 \mathrm{~K}$. Due to the uncertainties in the dust emissivity it is not easy to determine a total column density of dust (on the contrary, temperatures are almost independent on the dust emissivity). Nevertheless, the column density of dust with temperatures higher than $50 \mathrm{~K}$ is less than 500 times lower than the column of dust at $15-35 \mathrm{~K}$.
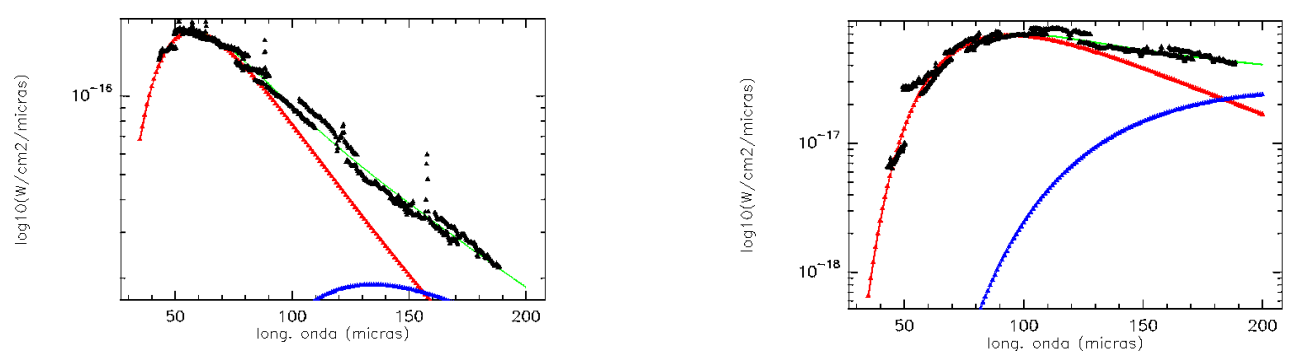

Fig. 1 Left panel: Dust continuum emission towards M+0.21-0.12 (black solid triangles) and grey-body fit (green) with two temperature components ar 39 (red) and $16 \mathrm{~K}$ (blue). Right panel: same for M+0.76-0.05 with a $24 \mathrm{~K}$ (red) and $15 \mathrm{~K}$ (blue) components. 
Table 1 Physical parameters derived from the observations. See text for explanation. Number in parentheses are errors of the last significant digit. Typical errors of $T_{1}$ and $T_{2}$ are 5 and $1 \mathrm{~K}$, respectively.

\begin{tabular}{lllllllllll}
\hline Source & $\begin{array}{l}n_{H_{2}} \\
\mathrm{~cm}^{-3}\end{array}$ & $\begin{array}{l}N_{H_{2}}(\mathrm{CO}) \\
\mathrm{cm}^{-2}\end{array}$ & $\begin{array}{l}N_{H_{2}} \\
\mathrm{~cm}^{-2}\end{array}$ & $\begin{array}{l}T_{H_{2}} \\
\mathrm{~K}\end{array}$ & $\begin{array}{l}L_{F I R} \\
\mathrm{~W} \mathrm{~cm}-2\end{array}$ & $\begin{array}{l}T_{1} \\
\mathrm{~K}\end{array}$ & $\begin{array}{l}T_{2} \\
\mathrm{~K}\end{array}$ & $\begin{array}{l}Q(H) \\
\mathrm{s}^{-1}\end{array}$ & $\begin{array}{l}n_{e} \\
\mathrm{~cm}^{-3}\end{array}$ & $\begin{array}{l}T_{\text {eff }} \\
\mathrm{K}\end{array}$ \\
\hline $\mathrm{M}-0.96+0.13$ & $3.5-4$ & $0.6-1.1$ & $1.10(9)$ & $157(6)$ & 2.9 & 30 & 15 & $\leq 47.8$ & $\leq 2.5$ & $\ldots$ \\
$\mathrm{M}-0.55-0.05$ & $3.8-4.4$ & $4.3-6.0$ & $2.7(3)$ & $135(5)$ & 12.3 & 31 & 16 & $\leq 47.5$ & $\leq 7.1$ & $\ldots$ \\
$\mathrm{M}-0.50-0.03$ & $3.4-3.7$ & $2.4-3.0$ & $2.3(2)$ & $135(4)$ & 10.5 & 34 & 17 & $\leq 48.0$ & $\leq 2.9$ & $\ldots$ \\
$\mathrm{M}-0.42+0.01$ & $4-4.5$ & $2.1-3.4$ & $1.03(8)$ & $167(6)$ & 9.8 & 30 & 16 & $\leq 48.0$ & $\leq 30$ & $\ldots$ \\
$\mathrm{M}-0.32-0.19$ & $>3$ & $1.1-2.2$ & $1.03(5)$ & $188(5)$ & 7.7 & 35 & 16 & $\leq 47.6$ & 4.4 & 34 \\
$\mathrm{M}-0.15-0.07$ & $3.7-4.1$ & $6.6-8.4$ & $2.6(4)$ & $136(6)$ & $\ldots$ & $\ldots$ & $\ldots$ & $\leq 47.6$ & $\ldots$ & $\ldots$ \\
$\mathrm{M}+0.16-0.10$ & $3.8-4.2$ & $3.7-4.9$ & $1.17(13)$ & $157(7)$ & 9.9 & 32 & 17 & $\leq 47.3$ & 9.4 & 35 \\
$\mathrm{M}+0.21-0.12$ & $>3$ & $0.8-1.5$ & $0.64(7)$ & $186(13)$ & 13.5 & 39 & 16 & $\leq 47.6$ & 15 & 35 \\
$\mathrm{M}+0.24+0.02$ & $3.5-3.9$ & $4.8-7.1$ & $1.73(6)$ & $163(2)$ & 16.1 & 30 & 16 & $\leq 47.4$ & 2.5 & $\ldots$ \\
$\mathrm{M}+0.35-0.06$ & $3.8-4.8$ & $1.7-2.7$ & $0.66(5)$ & $195(11)$ & 14.5 & 36 & 18 & $\leq 48.1$ & $\leq 20$ & $\ldots$ \\
$\mathrm{M}+0.48+0.03$ & $3.8-4.2$ & $3.2-3.6$ & $1.03(9)$ & $174(7)$ & 9.8 & 26 & 14 & $\leq 47.5$ & $\leq 16$ & $\ldots$ \\
$\mathrm{M}+0.58-0.13$ & $3.6-3.9$ & $3.1-3.9$ & $1.3(2)$ & $149(5)$ & 7.2 & 27 & 15 & $\leq 47.5$ & $\leq 2.5$ & $\ldots$ \\
$\mathrm{M}+0.76-0.05$ & $2.8-4.2$ & $6.6-8.6$ & $1.77(8)$ & $181(4)$ & 8.1 & 24 & 15 & $\leq 47.4$ & 11 & $\ldots$ \\
$\mathrm{M}+0.83-0.10$ & $3-4.5$ & $4.8-6.5$ & $1.59(6)$ & $178(5)$ & 6.3 & 26 & 15 & $\leq 48.0$ & $\leq 86$ & $\ldots$ \\
$\mathrm{M}+0.94-0.36$ & $>3$ & $1.3-2.9$ & $0.95(10)$ & $146(7)$ & $\ldots$ & $\ldots$ & $\ldots$ & $\leq 48.0$ & $\ldots$ & $\ldots$ \\
$\mathrm{M}+1.56-0.30$ & $3-4.5$ & $2-0.5$ & $0.26(10)$ & $260(30)$ & 1.5 & 27 & 17 & $\leq 48.0$ & $\leq 140$ & $\ldots$ \\
$\mathrm{M}+2.99-0.06$ & $3-4$ & $1.0-2.1$ & $1.40(9)$ & $152(3)$ & 1.7 & 29 & 18 & $\leq 48.0$ & $\leq 27$ & $\ldots$ \\
$\mathrm{M}+3.06+0.34$ & $3-3.8$ & $1.6-1.2$ & $0.21(8)$ & $250(20)$ & 1.1 & 34 & 18 & $\leq 48.0$ & $\leq 140$ & $\ldots$ \\
\hline
\end{tabular}

\section{Radio recombination lines}

To study the possible presence of ionized gas associated with the GCr molecular clouds we have observed the $\mathrm{H} 41 \alpha$ and $\mathrm{H} 35 \alpha$ recombination lines using the IRAM 30m antenna (RF03). However, we have not detected any of the lines in any of the sources. From our $3 \sigma$ limits to the line fluxes we have determined upper limits to the flux of Lyman continuum photons in the 30m beam (column 8 of Table 1). The comparison of those limits with stellar atmospheres models rules out the presence of stars of an earlier type than B0.5 and effective temperatures higher than $\sim 32000 \mathrm{~K}$.

\section{Fine structure lines}

We have detected lines of atoms and low excitational potential $(<13.6 \mathrm{eV})$ ions like O I $63 \mu \mathrm{m}, \mathrm{C}$ II 158 $\mu \mathrm{m}$ or Si II $34 \mu \mathrm{m}$ in all of the sources. In most of them we have also detected N II $122 \mu \mathrm{m}$ or Ne II 12 $\mu \mathrm{m}$ (excitational potential of 14 and $21 \mathrm{eV}$ respectively). In 11 of the 18 sources we have even detected the $\mathrm{O}$ III $88 \mu \mathrm{m}$ line (the excitational potential of $\mathrm{O}$ III is $35 \mathrm{eV}$ ). Column 9 of Table 1 shows the electron densities of the ionized gas component as derived from the O III 52 to O III $88 \mu$ m lines ratios. The densities, around 10-100 $\mathrm{cm}^{-3}$, are lower than those found in the Radio Arc region (RF01b) and in Sgr B2 (Goicoechea et al. 2003). Column 10 of Table 1 shows the effective temperatures derived from the $\mathrm{N}$ III $57 \mu \mathrm{m}$ to N II $122 \mu \mathrm{m}$ lines ratios for the three sources were the N III line has been detected. These 
temperatures have been calculated following the H II regions models by Rubin et al (1994). However, as pointed out by Shields and Ferland (1994) the observed lines ratios can even be reproduced with higher effective temperatures of the ionizing radiation if the ionization parameter is low, i.e., if the medium is clumpy and inhomogeneous and the ionizing sources are located far from the ionized nebulae. These seems to be the case of the Radio Arc region, were we have shown the presence of an extended component of gas ionized by the combined effect of the Quintuplet and the Arches clusters (RF01b). The radiation can reach large distances due to the inhomogeneity of the medium (in part due to the presence of a large bubble clearly seen in infrared images). Fotoionization model calculations showed that the lines ratios observed in this region can be explained with a constant effective temperature of the ionizing radiation but a different ionization parameter for each cloud consistently with the different distances of the clouds to the ionizing sources.

The analysis is more difficult for the clouds located far from the Radio Arc region since the geometry of the medium and the possible ionizing sources are unknown. However, fotoionization simulations for many lines ratios demonstrate that effective temperatures of $\sim 35000 \mathrm{~K}$ are possible if the ionization parameter is low $\left(\sim 10^{-3}\right.$, RF03).

Some of the fine structure lines have been observed in the Fabry-Perot mode. The spectral resolution of this mode $\left(\sim 30 \mathrm{~km} \mathrm{~s}^{-1}\right)$ give us the possibility of studying the line profiles of the broad GCr lines. Figure 2 shows a sample of the lines observed in this mode towards two sources. Taking into account the moderate spectral resolution, the lines profiles and the lines centers of highly excited ions like O III are in good agreement with those of the neutral or low excitational potential ions like (O I and C II). Furthermore, Fig. 3] shows that the agreement of the line profiles of the weakly ionized gas and the molecular gas is rather good. This fact suggest that the three components are associated and that radiation could play a role not only in the ionization of the ionized gas but also in the heating of the neutral gas.

We have compared the observed far infrared continuum and the C II, O I and Si II lines fluxes with the predictions from PDR models. The power radiated by lines is $\sim 0.5 \%$ of that radiated by the continuum. While the C II/O I ratio is $\sim 5$. Plotting both quantities in a plot like that of Fig. 2d of Goicoechea et al. (2003), one finds that the GCr clouds exhibit similar properties to those of Sgr B2, with a far ultra-violet field $\sim 10^{3}$ times larger than the local interstellar radiation field and a hydrogen density of $\sim 10^{3} \mathrm{~cm}^{-3}$. The absolute fluxes of the $\mathrm{C} \mathrm{II}\left(\sim 10^{-17} \mathrm{~W} \mathrm{~cm}^{-2}\right)$ and the $\mathrm{O}$ I line $\left(\sim 210^{-18} \mathrm{~W} \mathrm{~cm}^{-2}\right)$ are also consistent with the Hollenbach et al. (1991) PDRs models predictions.

\section{Discussion: heating and ionization}

The IRAM-30m "radio view" of the GCr interstellar medium seems to confirm the "classical" idea of a mainly neutral and dense gas. However, the global picture arising from the ISO observations is a complex interstellar medium with associated molecular, atomic, and ionized gas components with decreasing densities $\left(n_{H_{2}} \sim 10^{4}, n_{H} \sim 10^{3}, n_{e} \sim 10^{1-2} \mathrm{~cm}^{-3}\right.$ ). The ionizing radiation is hard (effective temperatures close to $\sim 35000 \mathrm{~K})$ but diluted due to the large distances from the ionizing sources to the nebulae $(\sim 50$ $\mathrm{pc}$ in the Radio Arc region). The large distance effect of the ionizing radiation can only be explained if the interstellar medium is very inhomogeneous. This scenario is also necessary to explain the low number of Lyman continuum photons derived from the radio recombination lines observations.

The radiation must also influence the atomic and molecular phases. As we have seen, the O I and C II lines are well explained by a PDR with a $\mathrm{H}$ density of $\sim 10^{3} \mathrm{~cm}^{-3}$ and a far ultra-violet incident field $10^{3}$ times higher than that in the local interstellar medium. The absolute $\mathrm{O}$ I and $\mathrm{C}$ II lines fluxes predicted by the PDR models are also similar the observed fluxes arising from the $\mathrm{GCr}$ clouds. Those models also predict a warm $\mathrm{H}_{2}$ layer with temperatures of $\sim 150 \mathrm{~K}$, as we have derived from the pure-rotational lines. However, the total column density of warm $\mathrm{H}_{2}$ predicted by the models is $\sim 310^{21} \mathrm{~cm}^{-2}$ while the total column density of warm $\mathrm{H}_{2}$ that we have derived from the pure-rotational lines is $\sim 10^{22} \mathrm{~cm}^{-2}$. Thus, 


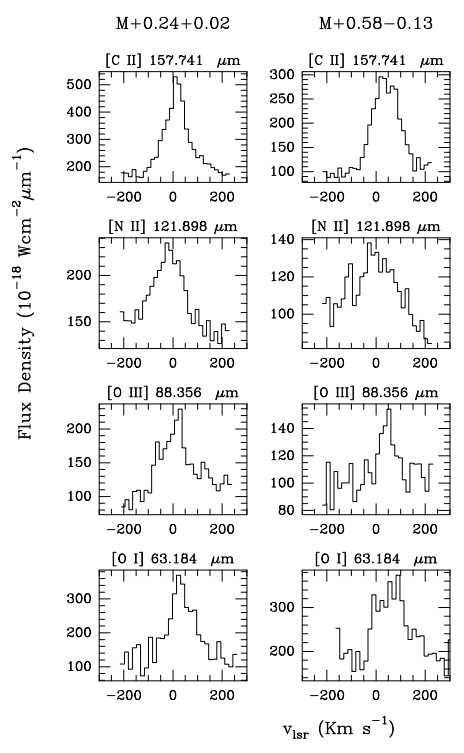

Fig. 2 Fabry-Perot spectra

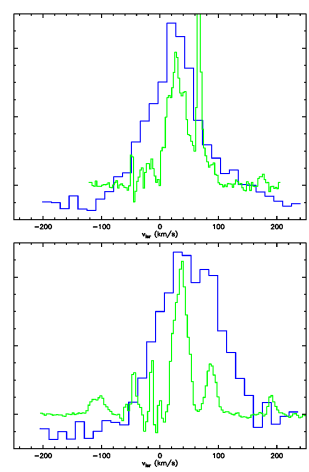

Fig. 3 A comparison of the line profiles of ${ }^{13} \mathrm{CO}(1-0)$ (green) and C II $158 \mu \mathrm{m}$ (blue) towards $\mathrm{M}+0.24+0.02$ (up) and $\mathrm{M}+0.58-0.13$ (down). Intensity in arbitrary units.

we conclude that approximately $30 \%$ of the warm molecular gas in the $\mathrm{GCr}$ clouds arises in PDRs in the external layers of the clouds.

It is important to note that the discrepancy of dust and gas temperatures only rules out gas heating by gas collisions with hot dust, but it does not rule out all radiative heating mechanisms. In the external layers of the proposed PDRs the gas is heated to temperatures of $\sim 150 \mathrm{~K}$ by photo-electric effect on the dust grains without heating the dust to temperatures higher than $\sim 35 \mathrm{~K}$ (Hollenbach et al. 1991). In fact, the $\sim 30 \mathrm{~K}$ dust component in the $\mathrm{GCr}$ can be associated to the $150 \mathrm{~K}$ gas.

At least a fraction of the other $70 \%$ of warm gas should be heated by shocks. The main evidence for shocks in the $\mathrm{GCr}$ are the high degree of turbulence revealed by the large line-widths and the high abundance in gas phase of molecules linked to the dust chemistry as $\mathrm{SiO}, \mathrm{NH}_{3}$ or $\mathrm{C}_{2} \mathrm{H}_{5} \mathrm{OH}$ (Martín-Pintado et al. 2001), which are easily photo-dissociated in the presence of ultra-violet radiation.

Acknowledgements NJR-F has has been supported by a Marie Curie Fellowship of the European Community program "Improving Human Research Potential and the Socio-economic Knowledge base" under contract number HPMFCT-2002-01677. NJR-F acknowledges useful discussions with J.R. Goicoechea.

\section{References}

Goicoechea, Rodríguez-Fernández and Cernicharo 2003, these proceedings

Hollenbach, D. J., Takahashi, T., \& Tielens, A. G. G. M. 1991, ApJ, 377, 192

Huettemeister, S., Wilson, T. L., Bania, T. M., \& Martin-Pintado, J. 1993,A\&A, 280, 255

Martín-Pintado, J., Rizzo, J. R., de Vicente, P., Rodríguez-Fernández, N. J., \& Fuente, A. 2001, ApJ, 548, L65

Rodríguez-Fernández, N. J., Martín-Pintado, J., Fuente, A., de Vicente, P., Wilson, T. L., \& Hüttemeister, S. 2001a, A\&A, 365, 174

Rodríguez-Fernández, N. J., Martín-Pintado, J., \& de Vicente, P. 2001, A\&A, 377, 631

Rodríguez-Fernández 2003 (in prep).

Rubin, R. H., Simpson, J. P., Lord, S. D., Colgan, S. W. J., Erickson, E. F., \& Haas, M. R. 1994, ApJ, 420, 772

Shields, J. C. \& Ferland, G. J. 1994, ApJ, 430, 236

Wilson, T. L., Ruf, K., Walmsley, C. M., Martin, R. N., Batrla, W., \& Pauls, T. A. 1982, A\&A, 115, 185 